\title{
Transgelin overexpression in lung adenocarcinoma is associated with tumor progression
}

\author{
XIAOHONG WU ${ }^{1}$, LIANGLIANG DONG ${ }^{1}$, RUIFENG ZHANG ${ }^{1}$, KEJING YING $^{1}$ and HUAHAO SHEN ${ }^{2}$ \\ ${ }^{1}$ Department of Respiratory Medicine, Sir Run Run Shaw Hospital, Zhejiang University School of Medicine, \\ Hangzhou, Zhejiang 310016; ${ }^{2}$ Department of Respiratory Medicine, Second Affiliated Hospital, \\ Zhejiang University School of Medicine, Hangzhou, Zhejiang 310009, P.R. China
}

Received February 14, 2014; Accepted June 3, 2014

DOI: $10.3892 /$ ijmm.2014.1805

\begin{abstract}
Hypoxia is a common feature of solid tumors and is associated with an increased likelihood of local recurrence and distant metastasis. Transgelin (TAGLN) is an actin crosslinking/polymerization protein that belongs to the family of actin-associated proteins, and there is evidence that TAGLN may be involved in the migration of epithelial cells by interacting with actin or promoting podosome formation. Cell migration is a key step of cancer metastatis. Thus, the aim of this study was to investigate the potential link between TAGLN protein levels and hypoxia in lung adenocarcinoma cells and to explore the possible functions and expression patterns of TAGLN in lung adenocarcinoma. We first examined the effects of altered TAGLN expression on cell migration under both normoxic and hypoxic conditions. Immunohistochemical (IHC) staining was also performed to examine TAGLN protein expression patterns in lung adenocarcinoma samples. Our results revealed that TAGLN was upregulated in the hypoxic lung adenocarcinoma cells. The inhibition of TAGLN expression in the cells using small interfering RNA (siRNA) led to a decreased migration ability. TAGLN was significantly overexpressed in the lung adenocarcinoma tissues compared to the adjacent tumor-free tissues. A high TAGLN expression correlated with an advanced TNM stage, lymph node metastasis and greater differentiation. TAGLN was upregulated in the human lung adenocarcinoma cell lines under hypoxic conditions, which contributed to
\end{abstract}

Correspondence to: Dr Kejing Ying, Department of Respiratory Medicine, Sir Run Run Shaw Hospital, Zhejiang University School of Medicine, 3 East Qingchun Road, Hangzhou, Zhejiang 310016, P.R. China

E-mail: yingkejing2014@126.com

Dr Huahao Shen, Department of Respiratory Medicine, Second Affiliated Hospital, Zhejiang University School of Medicine, 88 Jiefang Rd., Hangzhou, Zhejiang 310009, P.R. China

E-mail: Shenhh@163.com

Key words: lung adenocarcinoma, transgelin, hypoxia, migration, metastasis the migration ability of the cells. Thus, our data suggest that TAGLN may be a viable therapeutic target and a potential biomarker for predicting the prognosis of patients with lung adenocarcinoma.

\section{Introduction}

Lung cancer is both the most common cancer and the leading cause of cancer-related mortality in the majority of countries, including China. Metastatic lung cancer is responsible for $>90 \%$ of lung cancer-related deaths (1). Adenocarcinoma is the most common type of lung cancer, accounting for up to $30-40 \%$ of all cases. Approximately 60 to $70 \%$ of patients with lung adenocarcinoma already have a malignant pleural diffusion or distant metastasis at the time of diagnosis, with poor prognosis (2). Therefore, the notable rise in the incidence of lung adenocarcinoma and its lethal nature underscore the importance of understanding the complex metastatic process. Intensive efforts are underway to develop therapies to halt lung cancer metastasis.

Hypoxia, or areas of low oxygen levels, is a hallmark of solid tumors due to an imbalance between the oxygen supply and consumption. Hypoxic stress induces a variety of molecular responses through the activation of hypoxia-inducible factors (HIFs), which regulate a large number of genes that are exploited by tumor cells for several biological processes, including cell proliferation, apoptosis, immortalization and migration $(3,4)$. Accumulating clinical and experimental evidence has revealed a key role for intratumor hypoxia in promoting metastatic progression $(5,6)$. Although tumor hypoxia is a major therapeutic concern, the precise mechanisms of this process are poorly understood. It is hoped that a better understanding of hypoxia-associated proteins that may be involved in tumor progression and metastasis will lead to the identification of more effective therapeutic approaches (7).

Transgelin (TAGLN) belongs to the calponin family of actin-binding proteins, the members of which participate in diverse cellular processes, including cell motility and migration $(8,9)$. We have previously demonstrated that TAGLN contributes to the enhanced migration of hypoxic human pulmonary artery smooth muscle cells (hPASMCs) (10). In addition, TAGLN overexpression has been observed in gastric malignancies $(11,12)$ and pancreatic cancer $(13)$. Such 
interesting results led us to explore the largely unknown role of TAGLN in lung adenocarcinoma. In the present study, we examined TAGLN expression in the lung adenocarcinoma cancer cell lines, A549 and H358, following exposure to hypoxia. RNA interference (RNAi) was employed to evaluate the effects of TAGLN on cell migration under normoxic or hypoxic conditions. We also investigated the expression patterns and clinical significance of TAGLN protein levels in lung adenocarcinoma samples.

\section{Materials and methods}

Cell lines and cell culture. The human lung adenocarcinoma cell lines used in this study were originally obtained commercially from the Cell Bank of the Chinese Academy of Sciences (Shanghai, China). The cells were grown in RPMI-1640 medium supplemented with $10 \%$ heat-inactivated fetal bovine serum (FBS; Gibco/Invitrogen, Carlsbad, CA, USA), 100 IU/ml penicillin and streptomycin (Sigma-Aldrich, St. Louis, MO, USA) and incubated in an air $/ 5 \% \mathrm{CO}_{2}$ incubator set at $37^{\circ} \mathrm{C}$ in a humidified atmosphere. The cells were routinely passaged every 2-3 days when they reached approximately $80-90 \%$ confluence.

Cell treatment and protein extraction. An appropriate number of cells $\left(2 \times 10^{5}\right)$ was seeded into 6-cm dishes and exposed to hypoxia in a hypoxia incubator $\left(1 \% \mathrm{O}_{2}, 94 \% \mathrm{~N}_{2}\right.$ and $5 \% \mathrm{CO}_{2}$ ) for 12,24 or $48 \mathrm{~h}$. The incubator was sealed, and $\mathrm{N}_{2}$ was used to balance the reduced $\mathrm{O}_{2}$ level. Parallel cultures were placed in a normoxia incubator. Following incubation, whole-cell extracts were prepared by harvesting the cells and lysing them in radioimmunoprecipitation assay (RIPA) buffer [20 mM Tris- $\mathrm{HCl}, 137 \mathrm{mM} \mathrm{NaCl}, 1 \mathrm{mM} \mathrm{MgCl}_{2}$, $1 \mathrm{mM} \mathrm{CaCl}{ }_{2}, 10 \%$ glycerol, $1 \%$ NP-40, $0.5 \%$ deoxycholate, $0.1 \%$ sodium dodecyl sulfate (SDS) supplemented with $1 \mu \mathrm{g} /$ $\mathrm{ml}$ aprotinin, $1 \mu \mathrm{g} / \mathrm{ml}$ leupeptin, $0.57 \mathrm{mM}$ phenylmethanesulfonylfluoride (PMSF), $100 \mu \mathrm{M}$ sodium vanadate and $20 \mathrm{mM}$ $\beta$-glycerophosphate]. Following incubation on ice for $30 \mathrm{~min}$, the cells were scraped into a fresh tube, and the cell lysate was centrifuged $\left(12,000 \mathrm{rpm}, 20 \mathrm{~min}, 4^{\circ} \mathrm{C}\right)$ to remove cellular debris. The supernatant was harvested and stored at $-80^{\circ} \mathrm{C}$.

Western blot analysis. Protein concentrations of cell lysates were determined using the bicinchoninic acid (BCA) method using bovine serum albumin as a standard. Equivalent cell extracts (20-40 $\mu \mathrm{g}$ of protein) were resolved by $10 \%$ SDS-polyacrylamide gel electrophoresis. The protein was transferred onto a polyvinylidene difluoride (PVDF) membrane and blocked in Tris-buffered saline (TBS) containing 5\% non-fat dry milk and $0.2 \%$ Tween-20 for $1 \mathrm{~h}$. After blocking, the membrane was washed in TBS containing $0.2 \%$ Tween-20 3 times, and then probed with anti-TAGLN polyclonal antibody (Proteintech Group Inc., Chicago, IL, USA) at a dilution of 1:1,000 or anti-HIF monoclonal antibody (Abcam, Cambridge, UK) at a dilution of 1:1,000 at $4^{\circ} \mathrm{C}$ overnight, followed by incubation with appropriate horseradish peroxidase (HRP)-conjugated secondary antibodies. Proteins were detected using an enhanced chemiluminescence detection kit (Thermo Fisher Scientific, Inc., Waltham, MA, USA). An antibody to glyceraldehyde-3-phosphate dehydro- genase (GAPDH) was used as an internal loading control. Band intensities were analyzed using Quality One software (Bio-Rad, Hercules, CA, USA).

Inhibition of TAGLN expression by small interfering RNA (siRNA). TAGLN-specific siRNA and scrambled control siRNA were designed and synthesized by GenePharma (Shanghai, China). The sequence of TAGLN siRNA was as follows: forward, 5'-CCAAAAUCGAGAAGAAGUAdTdT-3' and reverse, 5'-UACUUCUUCUCGAUUUUGGdTdT-3'. The corresponding control siRNA sequence was: forward, 5'-UUC UCC GAA CGU GUC ACG U dtdt-3' and reverse, 5'-ACG UGA CAC GUU CGG AGA A dtdt-3'. Exponentially growing cells were plated in 6-well plates at an appropriate density and grown in antibiotic-free medium to $30-50 \%$ confluence prior to siRNA transfection. The cells were then transiently transfected with 100 pmolcontrol siRNA or TAGLN siRNA (final concentration of 33 nM RNA when added to the cells) using Lipofectamine 2000 reagent (Invitrogen) in serum-free RPMI-1640 medium for $6 \mathrm{~h}$ as described by the manufacturer's instructions. Briefly, 100 pmol siRNA and $5 \mu \mathrm{l}$ Lipofectamine 2000 per well were diluted separately in serum-free Opti-MEM to a final volume of $250 \mu \mathrm{l}$, gently mixed, and incubated at room temperature for $5 \mathrm{~min}$. Subsequently, the diluted siRNA solution and the diluted Lipofectamine 2000 were mixed gently and incubated at room temperature for $20 \mathrm{~min}$. The diluted siRNA/Lipofectamine 2000 complex was then added to the 6 -well plates containing 1,500 $\mu \mathrm{l}$ serum-free RPMI-1640 for $6 \mathrm{~h}$.

Transwell in vitro migration assay. The cells were grown in 6-well plates, transfected with 100 pmol TAGLN siRNA or control siRNA for $6 \mathrm{~h}$ and then cultured for a further $24 \mathrm{~h}$. The cells were trypsinized and resuspended in RPMI-1640 medium supplemented with $1 \%$ FBS. The cell suspension was adjusted to a concentration of $1 \times 10^{6} / \mathrm{ml}$ for the A549 cells or $2.5 \times 10^{6} / \mathrm{ml}$ for the H358 cells. Subsequently, $200 \mu \mathrm{l}$ cell suspension was seeded in the upper chambe220r of the Transwell (Corning Costar, Inc., Corning, NY, USA). The lower chamber was filled with $600 \mu \mathrm{l}$ RPMI-1640 medium containing 10\% FBS. After $24 \mathrm{~h}$ of incubation under normoxic or hypoxic conditions at $37^{\circ} \mathrm{C}$, the filter side of the upper chamber was cleaned with a cotton swab. The cells that had migrated across the filters were fixed for $5 \mathrm{~min}$ and stained for $20 \mathrm{~min}$ with Crystal violet solution (Sigma-Aldrich). The filter was gently cut from the chamber, and the number of migrated cells was counted in 4 high-power fields per insert (10x40). Three identical replicates were performed for each migration condition.

Wound healing assay. The cells were treated with 100 pmol TAGLN siRNA or control siRNA for $6 \mathrm{~h}$ and incubated for a further $24 \mathrm{~h}$ until confluent. Subsequently, the cell monolayer was manually scraped from one end of the well to the other with a sterile p200 pipet tip. The medium and cell debris were aspirated away and replaced with $2 \mathrm{ml}$ fresh serumfree medium. The cells were incubated under normoxic or hypoxic conditions, and their migration into the scratch area was monitored for up to $24 \mathrm{~h}$. Using a phase-contrast microscope, images of the scratch wound in the same field were captured 0 and $24 \mathrm{~h}$ after the wound was made. The relative width of the wound was measured quantitatively using Adobe 
Photoshop 5.0 (Adobe Systems Inc., San Jose, CA, USA) with the width at $0 \mathrm{~h}$ as the baseline. Wound closure (\%) was determined as the distance migrated after $24 \mathrm{~h}$ relative to the baseline width. Three identical replicates were performed for each migration condition.

Study population and sample preparation. A total of 75 patients who were diagnosed with lung adenocarcinoma between August 2006 to December 2008 were included in the current study. Matched tumor tissues and adjacent tumor-free tissues were obtained during surgery. Tissue samples to be used for immunohistochemistry (IHC) were fixed in formalin and embedded in paraffin. Patient clinicopathological data regarding gender, age, pathological TNM tumor stage, histology and grade were retrieved from the medical records. The study was approved by the hospital Ethical Committee, and written informed consent was obtained from all participants prior to enrollment.

IHC and scoring methods. The IHC detection of TAGLN was carried out with a lung adenocarcinoma tissue microarray containing matched duplicate tumor and para-cancerous tissue cores from the 75 patients. Briefly, $5-\mu$ m-thick microarray sections were deparaffinized in xylene and then rehydrated in a series of alcohols. Antigen retrieval was carried out by microwave treatment at $98^{\circ} \mathrm{C}$ for $10 \mathrm{~min}$ in $1 \mathrm{mmol} / \mathrm{l}$ ethylenediaminetetraacetic acid (EDTA, $\mathrm{pH}$ 8.0). The slide was then allowed to cool off at room temperature for $30 \mathrm{~min}$. Endogenous peroxidase activity was blocked in $3 \% \mathrm{H}_{2} \mathrm{O}_{2}$ in phosphate-buffered saline (PBS) for $30 \mathrm{~min}$ at room temperature. The slides were blocked with $10 \%$ bovine serum albumin/1X PBS at room temperature for $30 \mathrm{~min}$ to reduce non-specific background staining. Serum was removed from the slides, which were then incubated with mouse antiTAGLN monoclonal antibody $(1: 100)$ at $4^{\circ} \mathrm{C}$ overnight in a humidity chamber. The slide was then washed in PBS and incubated with biotinylated secondary antibodies for $30 \mathrm{~min}$ in PBS buffer, followed by 3 washes in PBS for 5 min each. Finally, the slides were incubated with biotinylated alkaline phosphatase-streptavidin (StreptABComplex/AP) for $20 \mathrm{~min}$ according to the manufacturer's instructions (Dako Denmark A/S, Glostrup, Denmark), developed with 3,3'diaminobenzidine (DAB) substrate, and counterstained with hematoxylin before mounting and light microscopy examination. Routine negative controls using non-immune serum instead of the primary antibody were included to verify the specificity. All immunoreactions were blindly evaluated by 2 independent experienced pathologists to quantify TAGLN protein expression. TAGLN immunoreactivity was scored on a 4-point scale as follows: negative, 0 ; weak, 1 ; intermediate, 2 ; and strong, 3. Cells with a staining intensity score of 1,2 or 3 were regarded as positive, while those with scores of 0 were regarded as negative. The percentage of positive tumor cells $(0 \%$, negative; $1-50 \%, 1 ; 51-75 \%, 2$; and $\geq 76 \%, 3)$ was assessed by counting $>1,000$ cancer cells in 10 randomly selected high-power fields (10x40). A combined staining score for each compartment was obtained as the product of intensity and extent of staining; a score of $<4$ was considered as a low expression, and a score of $\geq 4$ was considered as a high expression.
Statistical analysis. Statistical analyses were performed using SPSS version 13.0 for windows (SPSS Inc., Chicago, IL, USA). All results are presented as the means \pm standard error of the mean (SEM) from at least 3 independent experiments with similar results. Comparisons between 2 different groups were analyzed by using Student's t-tests. Correlations between TAGLN IHC scores and clinicopathological characteristics were evaluated using Fisher's exact tests. For all tests, a value of $p<0.05$ was considered to indicate a statistically significant difference.

\section{Results}

Hypoxia promotes TAGLN protein expression in lung adenocarcinoma cells. The A549 and H358 cells were exposed to normoxia $\left(5 \% \mathrm{O}_{2}\right)$ or hypoxia $\left(1 \% \mathrm{O}_{2}\right)$ for 12,24 or 48 . TAGLN protein expression showed no changes at $12 \mathrm{~h}$, but was markedly elevated in the A549 and H358 cells at $24 \mathrm{~h}$ compared with the corresponding normoxic controls. Its expression remained elevated for up to $48 \mathrm{~h}$ (Fig. 1).

SiRNA inhibition of TAGLN expression. In order to obtain the best silencing efficiency, $2 \times 10^{5}$ A549 cells/well or $5 \times 10^{5} \mathrm{H} 358$ cells/well were transfected with 100 pmol TAGLN siRNA (si-TAGLN) or control siRNA (si-control) using $5 \mu \mathrm{l}$ Lipofectamine 2000 on the basis of preliminary optimization experiments. At $48 \mathrm{~h}$ after transfection, the silencing effect at the protein level was determined by western blot analysis. si-TAGLN exhibited a potent silencing effect up to $70 \%$ compared with the negative control (Fig. 2).

Inhibition TAGLN of expression reduces lung adenocarcinoma cell migration. To explore the potential regulatory role of TAGLN in cancer cell migration, we performed wound healing and Transwell migration assays using the A549 and H358 cells under normoxic and hypoxic conditions. As shown by wound healing assay (Fig. 3), compared with the negative control, the cells transfected with si-TAGLN showed a slower wound healing rate, particularly under hypoxic conditions. As shown by Transwell assay, At $24 \mathrm{~h}$, the cells transfected with si-control had completely filled the gap with at least $80 \%$ wound closure under both conditions, while the cells transfected with TAGLN-specific siRNA showed 50\% wound closure at most. These results are consistent with those of the wound healing assay. As shown in Fig. 4, there were fewer migrated cells in the TAGLN-specific siRNA group than the negative control group. TAGLN-specific siRNA reduced the migration ability of the A549 cells by 28 and $39 \%$ under normoxic and hypoxic conditions, respectively. For the H358 cells, the percentages were 50 and $67.3 \%$, respectively. In addition, we found that the migration ability of the A549 and H358 cells improved after $24 \mathrm{~h}$ of exposure to hypoxia. Taken together, the results demonstrate that the decreased TAGLN expression suppressed A549 and H358 cell migration in vitro, particularly under hypoxic conditions. These findings indicate that TAGLN protein expression may play a key role in hypoxia-induced cell migration.

Correlation between TAGLN expression and clinicopathological characteristics. Representative results for TAGLN 
A
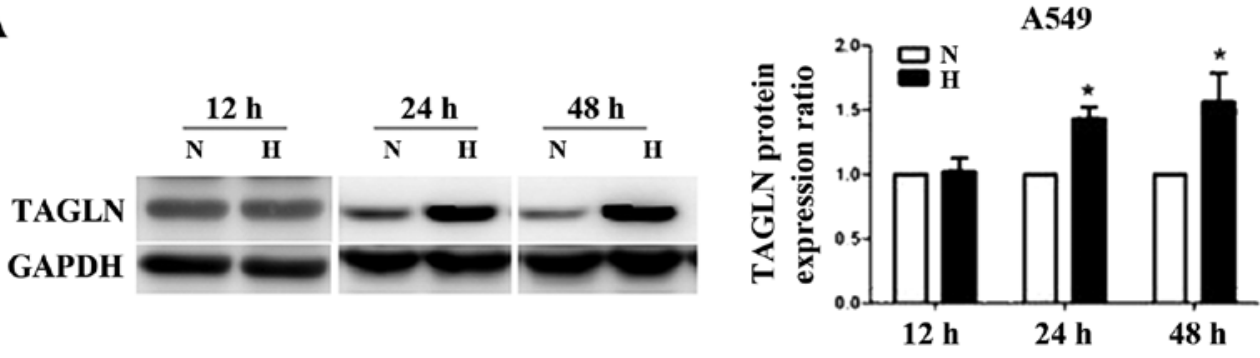

B
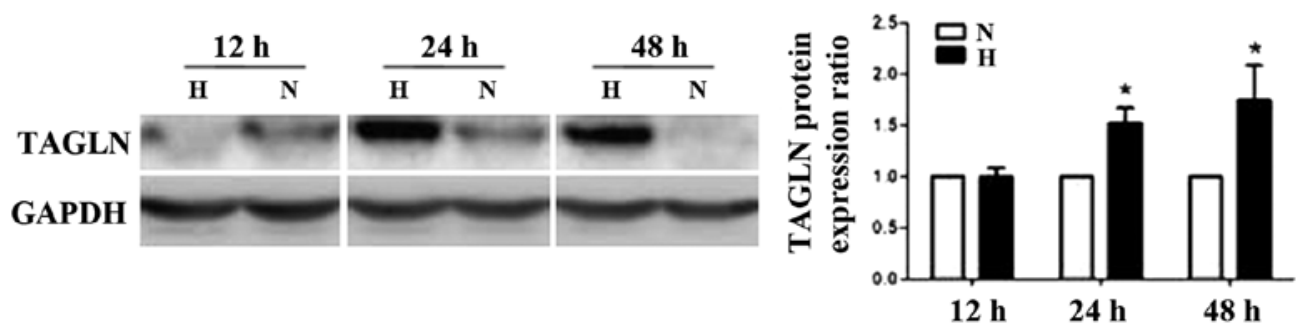

Figure 1. Increased transgelin (TAGLN) protein expression in lung adenocarcinoma cell lines under hypoxic conditions. Cells were exposed to either normoxia or hypoxia for 12, 24 or/and $48 \mathrm{~h}$ as indicated. TAGLN protein expression was detected by western blot analysis with glyceraldehyde-3-phosphate dehydrogenase (GAPDH) as a loading control. The ratios of relative TAGLN intensity measured in the hypoxic cells versus paired normoxic cells are shown for (A) A549 cells and (B) H358 cells, with the corresponding normoxic cells having a value of 1 . All assays were performed in triplicate, and the data are shown as the means \pm standard error of mean (SEM). ${ }^{*}$ p $<0.05$ compared with the normoxic control. N, normoxia; H, hypoxia.
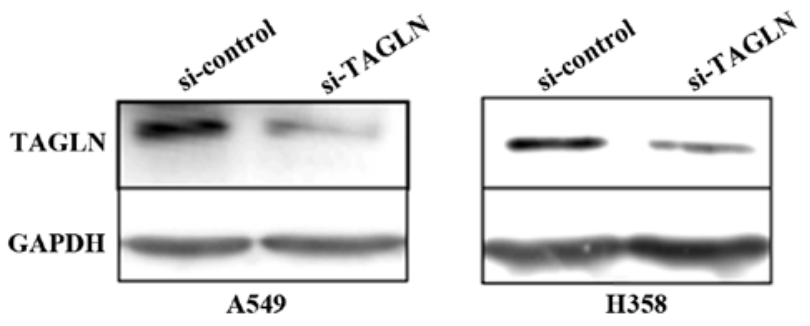

Figure 2. Inhibition of transgelin (TAGLN) protein expression using specific small interfering RNA (siRNA). Glyceraldehyde-3-phosphate dehydrogenase (GAPDH) served as a loading control.

protein expression in tumor tissue and adjacent tumor-free tissue are shown in Fig. 5. For the purpose of analysis, TAGLN IHC scores were classified as low $(<4)$ or high $(\geq 4)$. Using this classification, we detected significantly increased expression rates of TAGLN protein in tumor tissue $(51 / 75$, $68 \%)$ compared to the adjacent tumor-free tissue $(30 / 75$, $40 \%)\left(p=0.001, \chi^{2}=11.836\right)$. The association between TAGLN protein expression and the clinicopathological characteristics of the patients was investigated as shown in Table I. TAGLN expression was strongly associated with tumor stage $(\mathrm{p}=0.023$, $\left.\chi^{2}=6.117\right)$, lymph node status $\left(\mathrm{p}=0.025, \chi^{2}=5.672\right)$ and differentiation grade $\left(\mathrm{p}=0.014, \chi^{2}=6.63\right)$. A high TAGLN expression was observed in $83.9 \%(26 / 31)$ of the cases with advanced tumor stage and in $80 \%$ (32/40) of the lymph node-positive cases, while a low expression of TAGLN was observed in $55 \%(11 / 20)$ of the poorly differentiated cancers. No significant association was observed between TAGLN expression and other clinicopathologic characteristics, including gender, age and tumor size $(\mathrm{p}>0.05)$.

\section{Discussion}

Metastasis is a common phenomenon and the predominant cause of mortaltiy in patients with lung adenocarcinoma. There is growing evidence that hypoxia, which occurs in a wide range of solid tumors, is associated with a malignant tumor phenotype and augmented metastatic potential $(14,15)$. Hypoxia increases tumor angiogenesis, metastasis and other biological responses by activating the expression of relevant proteins through HIFs. It has previously been shown that each step of the metastatic process can potentially be regulated by hypoxia and the HIF system (16). Therefore, considerable research into the mechanisms of tumor metastasis has focused on hypoxia and hypoxia-associated proteins. We have previously reported that TAGLN protein expression is significantly induced by hypoxia in hPASMCs, which contributes to their increased motility under hypoxic conditions (10). With this in mind, we originally focused on the role of TAGLN in cancer. In the present study, we demonstrated that TAGLN is increased in lung adenocarcinoma cells under hypoxic conditions.

TAGLN, also known as SM22a, is a transformation- and shape change-sensitive actin stress fiber-binding protein that stabilizes actin gels (17). It was originally described as predominantly expressed in smooth muscle cells to bind to actin, suggesting its important roles in cytoskeletal rearrangement and the phenotypic modulation of cells $(18,19)$. Yu et al showed that increased TAGLN expression contributed to epithelial cell injury, repair, and migration in lung fibrosis (20). However, little is known about the potential functions of TAGLN in tumor progression. Therefore, in this study, we examined whether its expression contributes to the aberrant migration of lung adenocarcinoma cells, a prerequisite for tumor cell invasion and metastasis. Our experiments revealed that the inhibition of 
A

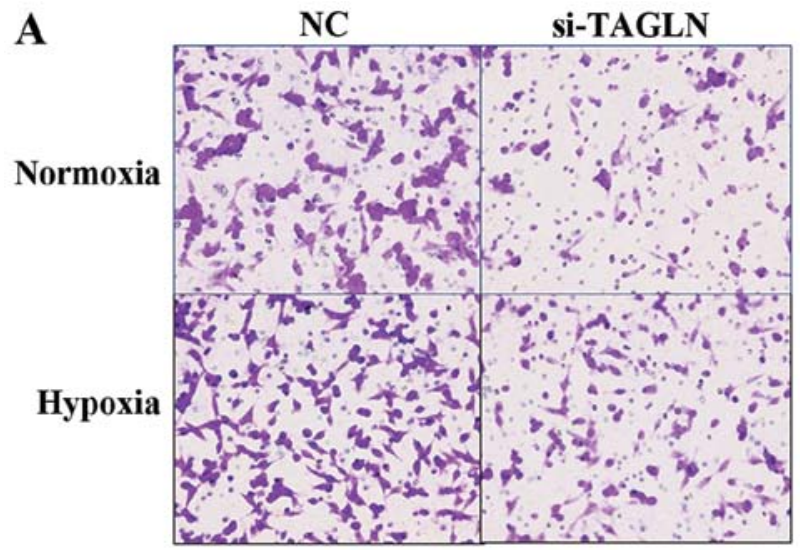

B

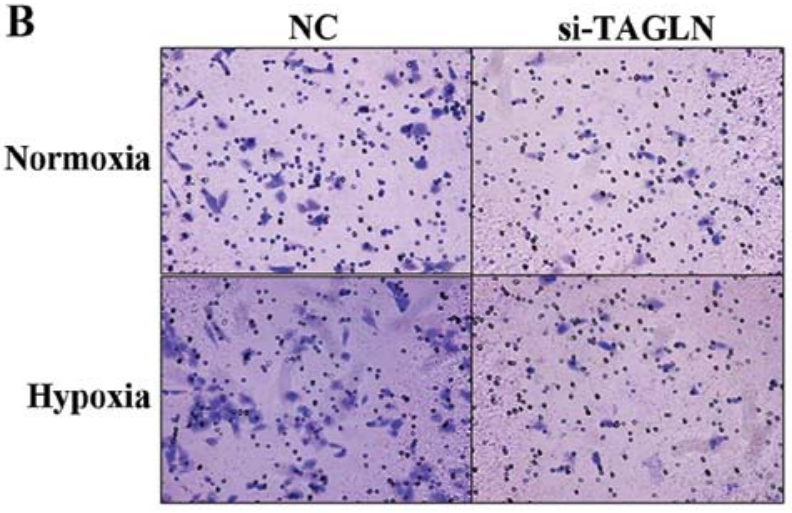

A549

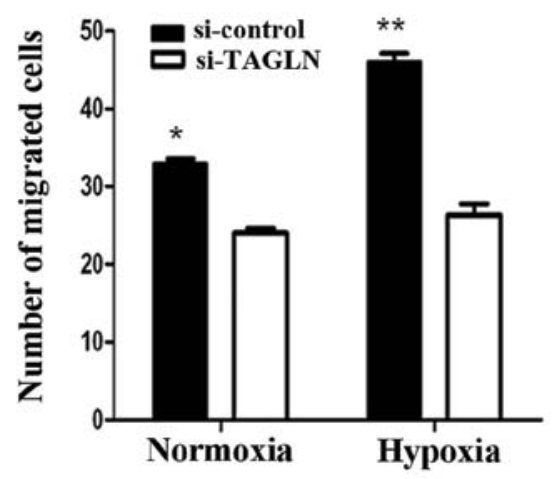

H358

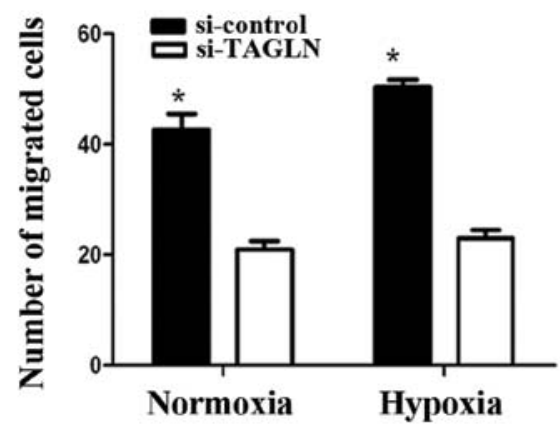

Figure 3. Effect of decreased transgelin (TAGLN) expression on wound healing under normoxic or hypoxic conditions. Cells seeded in 6-well plates were transfected with siRNA targeting TAGLN (si-TAGLN) or control siRNA (si-control) for $6 \mathrm{~h}$. Cell monolayers were wounded, and the gap closure was photographed to quantify the distance in 3 randomly selected sites per group. The wound closure (distance migrated after $24 \mathrm{~h}$ ) is expressed graphically. (A) Wound healing assay with the A549 cells. (B) Wound healing assay with the H358 cells. All assays were performed in triplicate, and data are shown as the means \pm standard error of mean (SEM). NC, si-control. ${ }^{*}<<0.05$ compared with the si-TAGLN, ${ }^{* *}$ p $<0.01$ compared with the si-TAGLN.

A
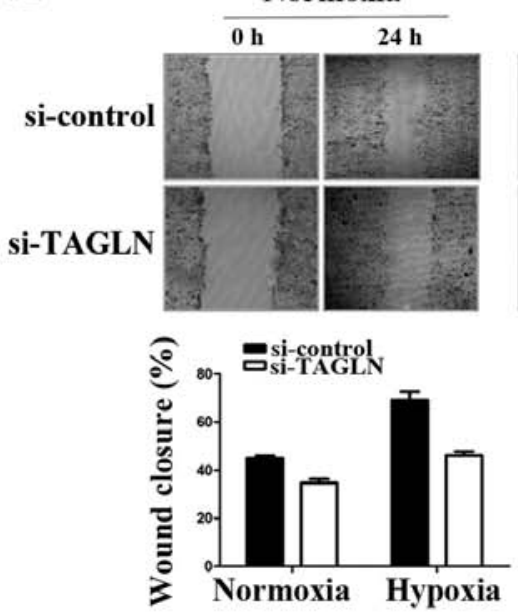

B
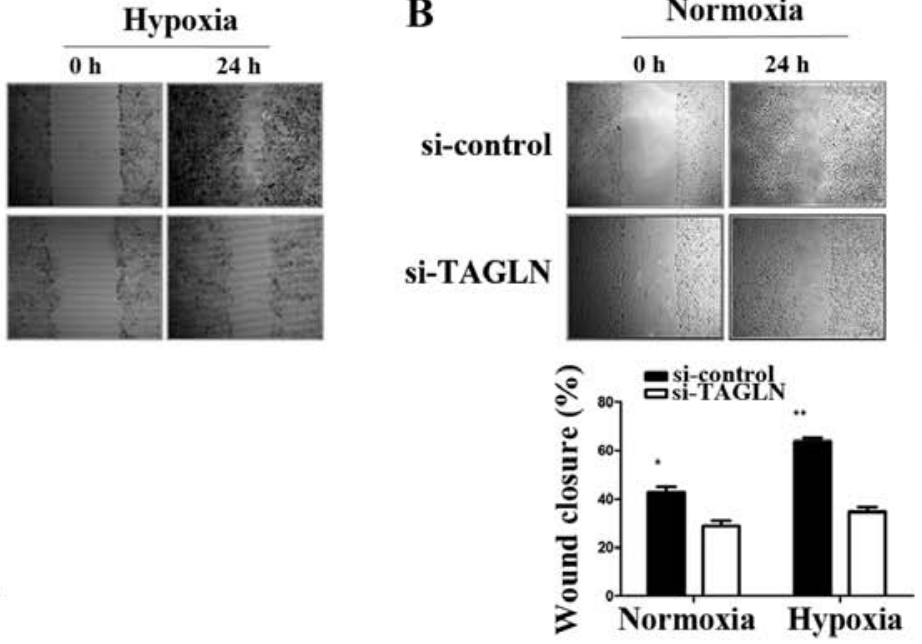

Figure 4. Effect of the inhibition of transgelin (TAGLN) expression on cell migration under normoxic or hypoxic conditions. Cells transfected with siRNA targeting TAGLN (si-TAGLN) or control siRNA (si-control) were plated into millicell inserts at a density of $2 \times 10^{5}$ cells/well for the A549 cells or $5 \times 10^{5}$ cells/ well for the H358 cells. Migrated cells were photographed and counted in 4 fields per insert after $24 \mathrm{~h}$ of incubation under normoxic or hypoxic conditions. (A) Transwell migration assay of A549 cells. (B) Transwell migration assay of H358 cells. All assays were performed in triplicate, and data are shown as the means \pm standard error of mean (SEM). ${ }^{*}<0.05$ compared with the si-TAGLN, ${ }^{* *}<<0.01$ compared with the si-TAGLN.

TAGLN expression using siRNA in A549 and H358 cells was accompanied by significantly impaired motility, particularly under hypoxic conditions. We also demonstrated that exposure to hypoxia stimulated A549 and H358 cell migration. Our motility data are suggestive of the potential role for TAGLN in lung adenocarcinoma cell migration and subsequent dissemi- 
Table I. Correlation between TAGLN expression and clinicopathological characteristics in lung adenocarcinoma.

\begin{tabular}{|c|c|c|c|c|c|}
\hline \multirow[b]{2}{*}{ Clinical classification } & \multicolumn{3}{|c|}{ TAGLN immunoreactivity } & \multirow[b]{2}{*}{$\mathrm{p}$-value } & \multirow[b]{2}{*}{$\chi^{2}$} \\
\hline & Total no. & Low & High & & \\
\hline \multicolumn{6}{|l|}{ Gender } \\
\hline Male & 41 & 13 & 28 & \multirow[t]{2}{*}{1 (Male vs. female) } & \multirow[t]{2}{*}{0.004} \\
\hline Female & 34 & 11 & 23 & & \\
\hline \multicolumn{6}{|l|}{ Age at surgery (years) } \\
\hline$\geq 60$ & 46 & 17 & 29 & \multirow{2}{*}{$0.313(\geq 60$ vs. $<60)$} & \multirow[t]{2}{*}{1.343} \\
\hline$<60$ & 29 & 7 & 22 & & \\
\hline \multicolumn{6}{|l|}{ T-primary tumor } \\
\hline $\mathrm{T} 1+\mathrm{T} 2$ & 55 & 19 & 36 & \multirow[t]{2}{*}{$0.578(\mathrm{~T} 1+\mathrm{T} 2$ vs. $\mathrm{T} 3+\mathrm{T} 4)$} & \multirow[t]{2}{*}{0.614} \\
\hline $\mathrm{T} 3+\mathrm{T} 4$ & 20 & 5 & 15 & & \\
\hline \multicolumn{6}{|l|}{ Lymph node status } \\
\hline Negative & 35 & 16 & 19 & \multirow[t]{2}{*}{0.025 (Negative vs. positive) } & \multirow[t]{2}{*}{5.672} \\
\hline Positive & 40 & 8 & 32 & & \\
\hline \multicolumn{6}{|l|}{ Stage } \\
\hline $\mathrm{I}+\mathrm{II}$ & 44 & 19 & 25 & \multirow[t]{2}{*}{0.023 (I + II vs. III + IV) } & \multirow[t]{2}{*}{6.117} \\
\hline III + IV & 31 & 5 & 26 & & \\
\hline \multicolumn{6}{|l|}{ Grade } \\
\hline Well + moderate & 55 & 13 & 42 & \multirow[t]{2}{*}{0.014 (Well + moderate vs. poor) } & \multirow[t]{2}{*}{6.63} \\
\hline Poor & 20 & 11 & 9 & & \\
\hline
\end{tabular}

TAGLN, transgelin.

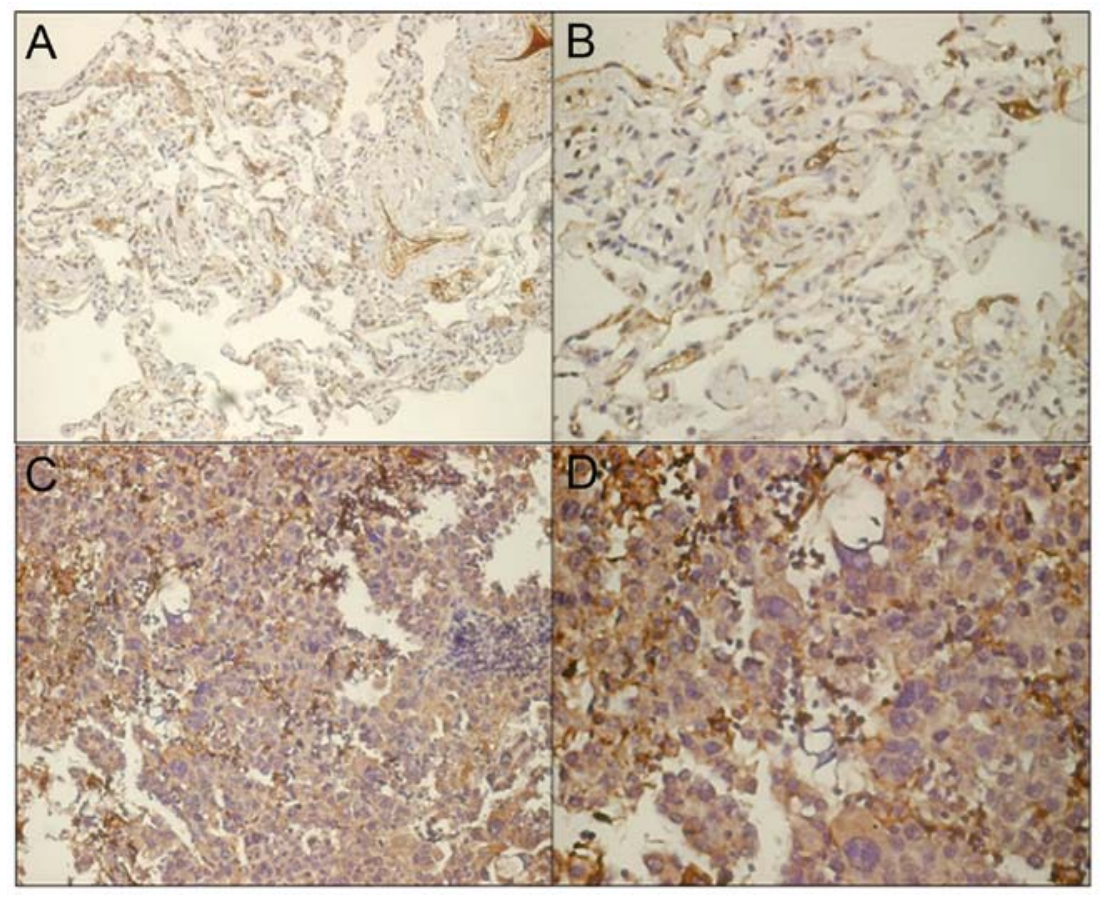

Figure 5. Transgelin (TAGLN) immunoreactivity in lung tissue. Serial sections of adjacent tumor-free tissues were stained with antibody to TAGLN and viewed at (A) x200 magnification and (B) x400 magnification. Serial sections of lung adenocarcinoma tissues were stained with antibody to TAGLN and viewed at (C) x200 magnification and (D) x400 magnification.

nation and metastasis. However, the underlying mechanisms are not yet fully understood. Gimona et al found that TAGLN is involved in podosome formation through association with a specific sub-population of actin filament bundles. They suggested that an increase in TAGLN expression may favor podosome formation by controlling the calponin/TAGLN 
ratio (21). Given the evidence that cell migration is triggered by stimulating intracellular signaling pathways that regulate actin cytoskeleton reorganization (22), we can infer that hypoxia or HIFs play a role in cancer cell migration and that TAGLN is involved in mediating the signaling pathways and can regulate the ability of cells to migrate through the direct interaction with the actin cytoskeleton. However, further studies are required to confirm this hypothesis.

As an actin-binding protein that controls cell motility, TAGLN may be valuable in assessing tumor progression or prognosis. The expression patterns of TAGLN have been reported to vary among different tumor types. It has been suggested that the loss of TAGLN expression is closely associated with progression, differentiation, metastasis and poor prognosis in colon cancer patients (23). In addition, the loss of TAGLN expression also occurs early in breast cancer progression, and TAGLN can inhibit prostate cancer cell growth $(8,24)$. These results suggest that TAGLN may act as a tumor suppressor. Indeed, the restoration of TAGLN expression both in vitro and in vivo inhibits carcinogenesis. This situation is reversed in other types of cancer; TAGLN expression is significantly increased in hepatocellular carcinoma (25), as well as gastric (11) and pancreatic cancer (26), suggesting its potential as a tumor biomarker for certain cancer types. These paradoxical roles of TAGLN in different forms of cancer reveal the diverse functions of TAGLN, and also led us to explore its expression patterns and clinical significance in lung adenocarcinoma.

We found that TAGLN was overexpressed in $>68 \%$ of the tested lung adenocarcinoma tissues compared to the paired adjacent tumor-free tissue. This overexpression did not correlate with the gender, age or tumor size of the patients. However, it was associated with TNM stage, lymph node status and differentiation grade. A higher TAGLN overexpression was found in cases with advanced TNM stages or lymph node-positive cases, which suggests that TAGLN is involved in malignant progression. Conversely, TAGLN expression was significantly lower in poorly differentiated tumors. Collectively, these results indicate that TAGLN may be a useful biomarker for tumor differentiation and for predicting lung adenocarcinoma prognosis.

In conclusion, to the best of our knowledge, the present study is the first to demonstrate that TAGLN is upregulated in human lung adenocarcinoma cell lines under hypoxic conditions, and this can enhance cellular migration ability. Compared to adjacent tumor-free tissues, lung adenocarcinoma tissues had significantly increased TAGLN immunoreactivity. Moreover, a higher TAGLN expression correlated with lymph node metastasis, TNM stage and differentiation. These results indicate that TAGLN may be a therapeutic target and a potential biomarker for predicting lung adenocarcinoma prognosis. However, further studies are required to fully elucidate the mechanisms involved.

\section{Acknowledgements}

This study was supported by a grant from the National Natural Science Foundation of China (no. 81000019).

\section{References}

1. Jemal A, Bray F, Center MM, Ferlay J, Ward E and Forman D: Global cancer statistics. CA Cancer J Clin 61: 69-90, 2011.
2. Silvestri GA, Alberg AJ and Ravenel J: The changing epidemiology of lung cancer with a focus on screening. BMJ 339: b3053, 2009.

3. Brahimi-Horn $\mathrm{C}$ and Pouysségur J: The role of the hypoxiainducible factor in tumor metabolism growth and invasion. Bull Cancer 93: E73-E80, 2006.

4. Fraga A, Ribeiro R and Medeiros R: Tumor hypoxia: the role of HIF. Actas Urol Esp 33: 941-951, 2009 (In Spanish).

5. Cassavaugh J and Lounsbury KM: Hypoxia-mediated biological control. J Cell Biochem 112: 735-744, 2011.

6. DeClerck K and Elble RC: The role of hypoxia and acidosis in promoting metastasis and resistance to chemotherapy. Front Biosci 15: 213-225, 2010.

7. Wilson WR and Hay MP: Targeting hypoxia in cancer therapy. Nat Rev Cancer 11: 393-410, 2011.

8. Assinder SJ, Stanton JA and Prasad PD: Transgelin: an actinbinding protein and tumour suppressor. Int J Biochem Cell Biol 41: 482-486, 2009.

9. Lambrechts A, Van Troys M and Ampe C: The actin cytoskeleton in normal and pathological cell motility. Int J Biochem Cell Biol 36: 1890-1909, 2004.

10. Zhang R, Zhou L, Li Q, Liu J, Yao W and Wan H: Up-regulation of two actin-associated proteins prompts pulmonary artery smooth muscle cell migration under hypoxia. Am J Respir Cell Mol Biol 41: 467-475, 2009.

11. Huang Q, Chen W, Wang L, Lin W, Lin J and Lin X: Identification of transgelin as a potential novel biomarker for gastric adenocarcinoma based on proteomics technology. J Cancer Res Clin Oncol 134: 1219-1227, 2008.

12. Ryu JW, Kim HJ, Lee YS, et al: The proteomics approach to find biomarkers in gastric cancer. J Korean Med Sci 18: 505-509, 2003.

13. Mikuriya K, Kuramitsu Y, Ryozawa S, et al: Expression of glycolytic enzymes is increased in pancreatic cancerous tissues as evidenced by proteomic profiling by two-dimensional electrophoresis and liquid chromatography-mass spectrometry/mass spectrometry. Int J Oncol 30: 849-855, 2007.

14. Arvelo F and Cotte C: [Hypoxia in cancer malignity. Review]. Invest Clin 50: 529-546, 2009 (In Spanish).

15. Subarsky P and Hill RP: The hypoxic tumour microenvironment and metastatic progression. Clin Exp Metastasis 20: 237-250, 2003.

16. Giatromanolaki A and Harris AL: Tumour hypoxia, hypoxia signaling pathways and hypoxia inducible factor expression in human cancer. Anticancer Res 21: 4317-4324, 2001.

17. Shapland C, Hsuan JJ, Totty NF and Lawson D: Purification and properties of transgelin: a transformation and shape change sensitive actin-gelling protein. J Cell Biol 121: 1065-1073, 1993.

18. Fu Y, Liu HW, Forsythe SM, et al: Mutagenesis analysis of human SM22: characterization of actin binding. J Appl Physiol 89: 1985-1990, 2000.

19. Shanahan CM, Weissberg PL and Metcalfe JC: Isolation of gene markers of differentiated and proliferating vascular smooth muscle cells. Circ Res 73: 193-204, 1993.

20. Yu H, Königshoff M, Jayachandran A, et al: Transgelin is a direct target of TGF-beta/Smad3-dependent epithelial cell migration in lung fibrosis. FASEB J 22: 1778-1789, 2008.

21. Gimona M, Kaverina I, Resch GP, Vignal E and Burgstaller G: Calponin repeats regulate actin filament stability and formation of podosomes in smooth muscle cells. Mol Biol Cell 14: 2482-2491, 2003.

22. Yamaguchi $\mathrm{H}$ and Condeelis $\mathrm{J}$ : Regulation of the actin cytoskeleton in cancer cell migration and invasion. Biochim Biophys Acta 1773: 642-652, 2007.

23. Zhao L, Wang H, Deng YJ, Wang S, Liu C, Jin H and Ding YQ: Transgelin as a suppressor is associated with poor prognosis in colorectal carcinoma patients. Mod Pathol 22: 786-796, 2009.

24. Yang Z, Chang YJ, Miyamoto H, Ni J, Niu Y, Chen Z, Chen YL, Yao JL, di Sant'Agnese PA and Chang C: Transgelin functions as a suppressor via inhibition of ARA54-enhanced androgen receptor transactivation and prostate cancer cell growth. Mol Endocrinol 21: 343-358, 2007.

25. Shi YY, Wang HC, Yin YH, et al: Identification and analysis of tumour-associated antigens in hepatocellular carcinoma. $\mathrm{Br}$ J Cancer 92: 929-934, 2005.

26. Zhou L, Zhang R, Zhang L, Sun Y, Yao W, Zhao A, Li J and Yuan Y: Upregulation of transgelin is an independent factor predictive of poor prognosis in patients with advanced pancreatic cancer. Cancer Sci 104: 423-430, 2013. 